CERN-PPE/92-53

20 March 1992

\title{
High Flux Operation of Microstrip Gas Chambers on Glass and Plastic Supports
}

R.Bouclier, J.J.Florent, J.Gaudaen, G.Millon, A.Pasta, L.Ropelewski, F.Sauli, L.I.Shekhtman*

CERN, Geneva, Switzerland

\begin{abstract}
Recent observations on Microstrip Gas Chambers realized on various glass and plastic supports are presented in this paper. Short term measurements indicate a rate capability up to and above $510^{5}$ counts $/ \mathrm{s} . \mathrm{mm}^{2}$. A long term exposure to radiation shows however gain modifications, dependent on the resistivity of the chamber substrate; choice of low resistivity supports minimizes this effect.
\end{abstract}

Presented by L. Ropelewski at the

Sixth International Wire Chamber Conference

Vienna, February $17-21,1992$

* on leave of absence from BINP Novosisbirsk, Russian Federation 


\section{Introduction}

Microstrip Gas Chambers (MSGC) are a very promising new type of position sensitive gas detectors intrinsically capable to overcome some limitations of existing multiwire chambers. MSGCs are realized with thin metal anode and cathode strips printed on insulating supports using photo- or electronlithography techniques. Very small spacings between electrodes can be realized, substantially reducing the drift distance for collection of positive ions produced in the avalanches, thus decreasing space charge effect and increasing the rate capability. A high signal to noise ratio and the radiation hardness of amorphous materials used in construction give an advantage to MSGC as compared to silicon detectors.

Proportional gains above $10^{4}$, position accuracy around $30 \mu \mathrm{m}$ and very good energy resolution $(11 \%$ at $5.9 \mathrm{keV})$ have been demonstrated in the early works [1-8]. Various groups reported however [9-11] instabilities and gain modifications that could be attributed to the charging up of the insulating surface between electrodes. This can possibly be avoided by reducing the surface resistivity of the support by ion implantation [7-8]. This method imposes however a constraint on the maximum size of the detector and tends to be expensive. One may achieve the same result using supports with a small bulk conductivity.

We have developed and tested detectors on several glass and plastic supports in a wide range of bulk resistivities. Glass is best suited for standard photolithography because of its good surface quality, and allows construction of large structures because of its mechanical stability. On the other hand, glass substrata less than $0.5 \mathrm{~mm}$ thick are difficult to handle. Microstrip chambers can be made on plastic supports, $100 \mu \mathrm{m}$ thick or less, having moreover low $\mathrm{Z}$ and in addition enabling non-planar geometry because of flexibility $[6,12,13]$. The wide range of existing commercial plastics with different resistivities may help to solve the problem of the surface charging up. Due to the limited resistance to heating and poor surface quality however plastics require more careful treatment during chamber fabrication and in general yield a lower quality product.

We have also developed a computer program [14] that allows to calculate the electric fields in the MGSC in the presence of a resistive support, and used it for better understanding the general operating properties of the detector and to optimize its performances.

This paper reports some preliminary results obtained with MSGC realized on glass and plastic supports exposed to high flux and for both short and long term irradiation.

\section{Experimental Set-up}

We have built and tested several chambers realized on soda lime glass 1 supports $1 \mathrm{~mm}$ thick and with a nominal bulk resistivity of $10^{15} \Omega \mathrm{cm}$. At the same time in collaboration with specialized laboratories ${ }^{2}$ we have obtained samples of special glasses with lower resistivity ranging from $10^{9}$ to $10^{12} \Omega \mathrm{cm}$. The MSGC patterns were realized by micro-lithography followed by chemical etching of an evaporated layer of chromium ${ }^{3}$ or aluminium $40.3 \mu \mathrm{m}$ thick in

\footnotetext{
1 Hoya SL

2 Murano, Stazione Sperimentale del Vetro, Venezia, Italy Institute of Physics, Technical University of Gdansk, Gdansk, Poland

Scientific Research Institute of Electrovacuum Glass, Moscow, Russian Federation

3 Artwork by Baumer IMT, Industrielle Messtechnik AG, Greifensee, Switzerland

4 Artwork by Thin Film \& Glass and Printed Circuit groups at CERN
} 
case of special glasses and plastics. Anode and cathode strips alternated on the substratum with a distance of $200 \mu \mathrm{m}$ between centers with a width of 10 and $50 \mu \mathrm{m}$ respectively for glass, 30 and $150 \mu \mathrm{m}$ for plastic. We have also tested a chamber with gold electrodes 5 having $3 \mu \mathrm{m}$ wide anodes, $60 \mu \mathrm{m}$ wide cathode strip widths and $100 \mu \mathrm{m}$ distance between centers of electrodes realized on a glass support with a bulk resistivity of $10^{9} \Omega \mathrm{cm}$. A schematic cross section of the typical chamber realized on a thin plastic support is shown in Fig. 1 and the corresponding computed electric field lines in the region close to the strips in Fig. 2.

The choice of a $400 \mu \mathrm{m}$ anode to anode pitch for the glass chambers, coarser than the one used in most previous works, was determined by the intention to allow comparison with the plastic chambers, where we were restricted by the resolution limits in the production facility. Consequently one can expect an enhancement of the charging up process due to the wider regions of insulator between electrodes. Recently, we have obtained samples of plastic chambers with improved strips quality and $200 \mu \mathrm{m}$ pitch 6 . These chambers are currently being tested.

We have found so far two plastic supports, Kapton and Tedlar ${ }^{7}$, suitable for fabrication of MSGC, being mechanically stable, with good surface quality and having sufficiently strong adherence of the vacuum evaporated metal layer. The bulk resistivity of these plastics is around $10^{17}$ and $10^{14} \Omega \mathrm{cm}$ respectively.

We have tested chamber prototypes with sensitive area of $40 \times 10$ and $100 \times 30 \mathrm{~mm}^{2}$. The chambers' frames were constructed with standard materials like epoxy, G10 and mylar. In all tests, groups of anodes of 20 to 30 strips were connected together to a low noise charge amplifier; unless otherwise mentioned, the measurements were performed using an argonmethane 90-10 gas mixture at atmospheric pressure. The choice of this gas was dictated by convenience for reproducibility of results, and easy determination of the parameters needed for computer simulations of the drift and avalanche processes. As a radiation source we used either $5.9 \mathrm{keV} \mathrm{X}$-rays from ${ }^{55} \mathrm{Fe}$ or $9 \mathrm{keV}$ photons from $\mathrm{X}$-ray tube with copper anti-cathode for the high flux tests.

\section{Results}

Fig. 3 shows the dependence of proportional gain on the anode voltage with the cathode strips grounded and all other potentials fixed for Hoya SL glass support. One can see that gains close to $10^{4}$ could be safely reached. A typical pulse height spectrum for $5.9 \mathrm{keV}$ X-rays is shown in Fig. 4 and has an energy resolution better than $20 \%$ fwhm. Both measurements were performed at low rates (less than 100 counts $/ \mathrm{s} \cdot \mathrm{mm}^{2}$ ).

The main problem connected with the operation of the MSGC appears to be the stability of the gain for long irradiation periods (see Fig. 5). One can see that even for moderate rates there is a substantial drop of the gain during the first few hours of operation of the chamber on the SL glass support. This drop doesn't depend on the rate and has a global nature, i.e. it is not localized around the irradiation spot. After switching off the high voltage for a few hours the gain restores to its initial value.

One possible explanation for the drop is the nature of the conductivity in glass, in most common glasses due to the motion of alkali ions. After applying a difference of potential, ions migrate in the bulk from one electrode to the other

\footnotetext{
5 Artwork by BINP Novosibirsk, Russian Federation.

6 Produced by PPM Co., Montreal, Canada

7 Trade names of DuPont de Nemours Co.
} 
and since there is no fresh source of ions, the number of current carriers decreases resulting in the increase of the resistivity with time (see Fig. 5). An indication of ion conductivity is the non-proportionality of conduction current to supplied voltage. On the contrary, an ohmic behavior in the glass suggest an electronic mechanism of conduction, and exhibits constant resistivity with time. Fig. 6 shows the measured time dependence of the resistivity for several commercial glasses compared with special glasses having electronic conductivity. A comparison of the time dependence of gain for two types of glasses is shown in Fig. 7. A chamber built on the electronically conducting glass doesn't show gain changes during the initial operation. Although we found commercially available glass (Corning 0317) with an ohmic behavior and suitable bulk resistivity, we never have been able to operate the chamber on this glass due to the large noise signal.

The other problem we found operating the chambers is connected with surface charging up. Fig. 8 shows the measured gain dependence on rate of the SL glass chamber for different operating voltages; curve 1 corresponds to the computed optimal voltage setting, where the field lines do not cross the surface of the insulator. In other cases (curves 2 and 3 ) the probability of the collection of ions from the gas on the surface of the glass is higher resulting in modification of the electric field and a drop of the gain at increasing rate. Note that in this case the gain doesn't restore to its initial value after reducing the rate. The importance of applying an appropriate positive potential to the back plane electrode to reduce the modification of the gain due to the charging up of the surface of the plastic chamber was already reported $[1,12,13]$.

Fig. 9 shows the effect of space-charge on the gain. The measurement was performed by exposing the Tedlar MSGC to increasing fluxes for a very short time to avoid the appearance of surface charging up. As one can see, a gain drop is observed only at rates approaching $10^{6}$ counts $/ \mathrm{s} . \mathrm{mm}^{2}$, a very good performance when compared with traditional MWPC. The reduction of space charge effect is a consequence of the fact that a large fraction of positive ions produced in the avalanches are collected very quickly by the cathode strips very close to the anodes. This fraction can be controlled by tuning the potential of the drift electrode [14].

We have studied the charging up effects measuring the long term variations of gain of the chambers exposed to constant high fluxes. Fig. 10 shows a comparison of the time behavior for three chambers constructed on different glass supports with resistivities covering a range of 6 orders of magnitude. A stable operation was achieved with the chamber manufactured on electronic glass with resistivity of $10^{9} \Omega \mathrm{cm}$. We haven't noticed any gain modifications after irradiation equivalent to approximately $2.5 \mathrm{mC}$ of charge collected per $\mathrm{cm}$ of strip. As mentioned above this chamber had a smaller pitch $(200 \mu \mathrm{m})$ and gold electrodes; we do not know the relevance of this difference on the result. However it's known that chromium and aluminium are chemically more active than gold, they form protective oxide layer when used as electrode material [15] leading to Malter-like breakdown effects.

All other chambers show a continuous drop of the gain accompanied by a degradation of the energy resolution. The rate of decrease of the gain depends on the flux, as can be seen in Fig. 11. We found that the gain modification due to irradiation is very localized to the region of the beam spot, and shows long term memory indicating the influence of other factors than surface charging up (that one would expect to disappear due to conductivity). Indeed after examination of the irradiated region under the microscope we have found deposits on the anode strips that could be cleaned up, a well known phenomenon encountered in aging of gaseous detectors. We have tested several gas mixtures believed to reduce aging processes [15] (see Fig. 12) and did not notice any improvement of the performance of the chamber. In all cases except the mixture of argon-DME (93-7) we found visible deposits on the 
anode strips after irradiation. Knowing that water can have a dramatic influence on the behavior of the chambers due to the fact that a change in the relative humidity by $20 \%$ results in change of the surface resistivity of the glass by 3 orders of magnitude, we have added small amount of water vapor to the gas in the chamber for a short period of time. After terminating water vapor flow we have observed restoration of the gain for a short time, without however improving a degraded energy resolution.

It has been already demonstrated $[13,14]$ that gaseous microstrip chambers realized on thin plastic supports can be operated at large proportional gains with relatively good energy resolution. These chambers show similar rate dependent gain modification due to the surface charging up, more pronounced for higher resistivity chamber supports; in Fig. 13 we show the results obtained for the Tedlar support (bulk resistivity $\sim 10^{14} \Omega \mathrm{cm}$ ). At moderate rates the gain is constant or even slightly increasing. If one keeps irradiation time short enough to prevent the appearance of aging, the gain returns to its original value after removal of the source (see Fig. 14) providing some evidence for a surface charging up effect.

\section{Conclusions and Summary}

We succeeded in building and operating microstrip gas chambers on several glass and plastic supports. We have identified four effects of different importance affecting the performance of the MSGC detectors at high fluxes:

- depletion of ionic carriers under application of difference of potentials, a consequence of the nature of ionic conductivity in glass, results in an increase of resistivity of the glass support and gain drop of the detector in first few hours of operation. It can be avoided by the proper choice of a substratum having only electronic conductivity.

- accumulation of charges produced in the avalanches on the surface of insulator leading to a rate dependent, dynamic modification of gain. It can be avoided by lowering the surface resistivity of the support (ion implantation, thin film deposit) or by decreasing the bulk resistivity. This effect can be also reduced substantially by a proper choice of the operating conditions, particularly of the back plane potential.

- accumulation of positive ions in the gas volume (space charge effect) that results in decrease of the gain. This is an intrinsic limitation of gaseous counters determined by the geometry of the detector, that can be minimized by a proper choice of the electrode potentials.

- aging: our experience shows that aging properties (deposit and/or permanent strip damage) of MSGC are comparable to those observed in other gaseous detectors; one should carefully select materials and gas mixtures used for operation of the microstrip gas detectors.

\section{Acknowledgements}

The authors would like to thank B. Bencivenni from INFN, Frascati and Yu. Pestov from BINP, Novosibirsk for their help in arranging the production of the samples of special glass and $M$. Dixit from Carleton University, Montreal for organizing the fabrication of Tedlar chambers. 


\section{References}

[1] A.Oed et al, Nucl. Instr. Methods A263 (1988) 351.

[2] F. Angelini et al, Nucl. Instr. Methods A283 (1989) 755.

[3] H. Hartjes and F. Udo, Proc. ECFA Study Week on Instr. Techn. for High Luminosity Hadron Colliders, Barcelona 1989, CERN 89-10 (1989) 455.

[4] F. Angelini et al, Proc. Large Hadron Collider Workshop, Aachen (1990), CERN 90-10 (1990) 222.

[5] F. Angelini et al, Nucl. Physics B 23A (1991) 254.

[6] H. Stahl et al, Nucl Instr. Methods A297 (1990) 95.

[7] F. Angelini et al, CERN-PPE 91-122.

[8] F. Angelini et al, INFN PI/AE 91/10.

[9] R.A. Lewis et al, Proc. European Workshop on X-Ray Detectors for Synchrotron Radiation Sources, Aussois 1991.

[10] C. Budtz-Jorgensen et al, Proc. European Workshop on X-Ray Detectors for Synchrotron Radiation Sources, Aussois 1991.

[11] J.E. Bateman et al, Proc. European Workshop on X-Ray Detectors for Synchrotron Radiation Sources, Aussois 1991, RAL-91-076.

[12] R. Bouclier et al, Proc. $5^{\text {th }}$ Pisa Meeting on Advanced Detectors, Pisa 1991, CERN-PPE 91-108.

[13] R. Bouclier et al, Proc. IEEE Nuclear Science Symposium, Santa Fe 1991, CERN-PPE 91-227.

[14] J.J. Florent et al, The electric field in microstrip gas chambers and its influence on detector performance, to be published.

[15] J.A. Kadyk, Nucl. Instr. Methods, A300 (1991) 436. 


\section{Figure Captions}

Fig. 1: Schematic cross section of a thin plastic microstrip chamber.

Fig. 2: Field lines calculated for the chamber realized on thin plastic support, for optimized potentials.

Fig. 3: Proportional gain dependence on the anode voltage for Hoya SL glass chamber.

Fig. 4: Pulse height spectrum measured with $5.9 \mathrm{keV}{ }^{55} \mathrm{Fe} \mathrm{X}$-ray source for the Hoya SL glass chamber.

Fig. 5: Initial gain variation as a function of time after applying the voltage for the Hoya SL glass chamber compared with the change of the resistivity of the support glass.

Fig. 6: Measured time dependence of the resistivity for several commercial glasses (Hoya SL, Pyrex, Corning 0317) compared with that for special glasses having electronic conductivity (Moscow, Murano).

Fig. 7: Comparison of the initial time dependence of the gain for the Hoya SL glass and Murano glass chambers.

Fig. 8: Measured gain dependence on rate of the SL glass chamber for three different operating voltages. Curve 1 corresponds to the computed optimal voltage setting, where the field lines do not cross the surface of the insulator (see Fig. 1).

Fig. 9: Gain dependence on the rate measured for the Tedlar chamber with the optimized operational voltages; measurement time was short enough (several seconds) to neglect surface charging up and aging effects.

Fig.10: Gain variation as a function of time for three different chambers realized on the supports covering resistivity range of 6 orders of magnitude. The total collected charge at the end of measurement corresponds to $1.5 \mathrm{mC}$ per $\mathrm{cm}$ of strip.

Fig.11: Time dependence of the gain measured for the Murano glass chamber with three different $X$-ray fluxes at fixed operational voltages.

Fig.12: Time variation of the gain for the Murano glass chamber filled with argon-ethane gas mixture with addition of three different vapors measured at fixed voltages and $X$-ray flux.

Fig.13: Gain modification as a function of time for the Tedlar chamber for different radiation rates.

Fig.14: Gain recovery observed in the Tedlar chamber. After 6 hours of irradiation with the flux of $10^{3} \mathrm{c} / \mathrm{s} \cdot \mathrm{mm}^{2}$, the rate was reduced by two orders of magnitude. 


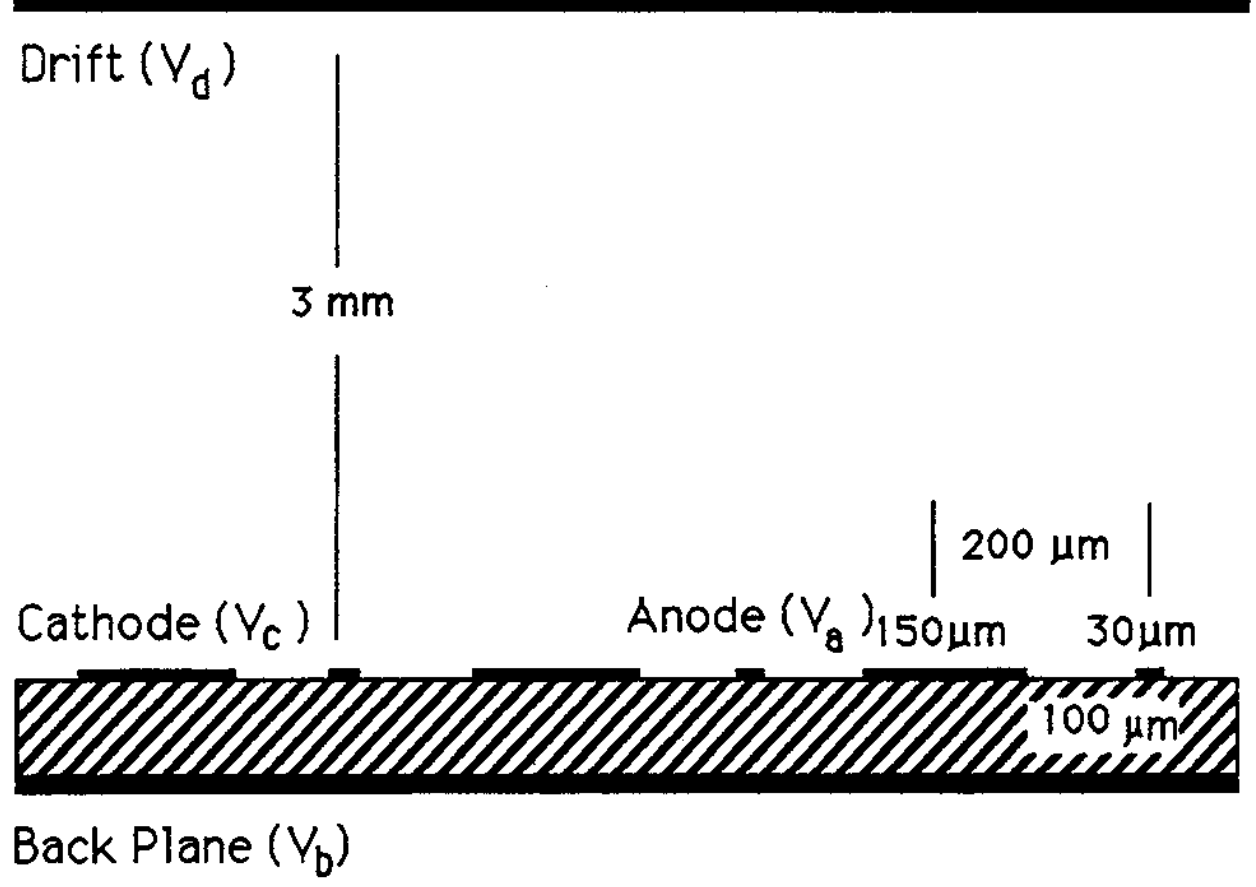

Fig. 1

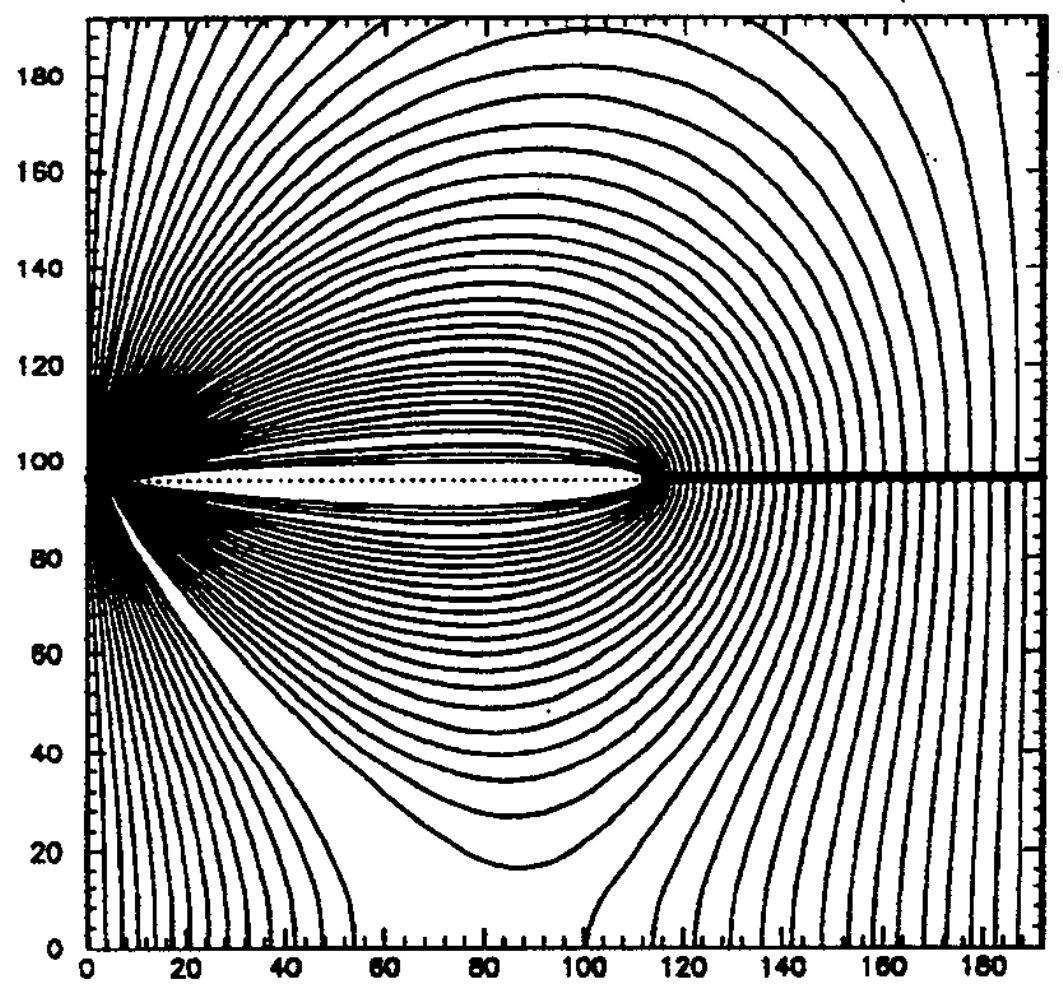

Fig. 2 


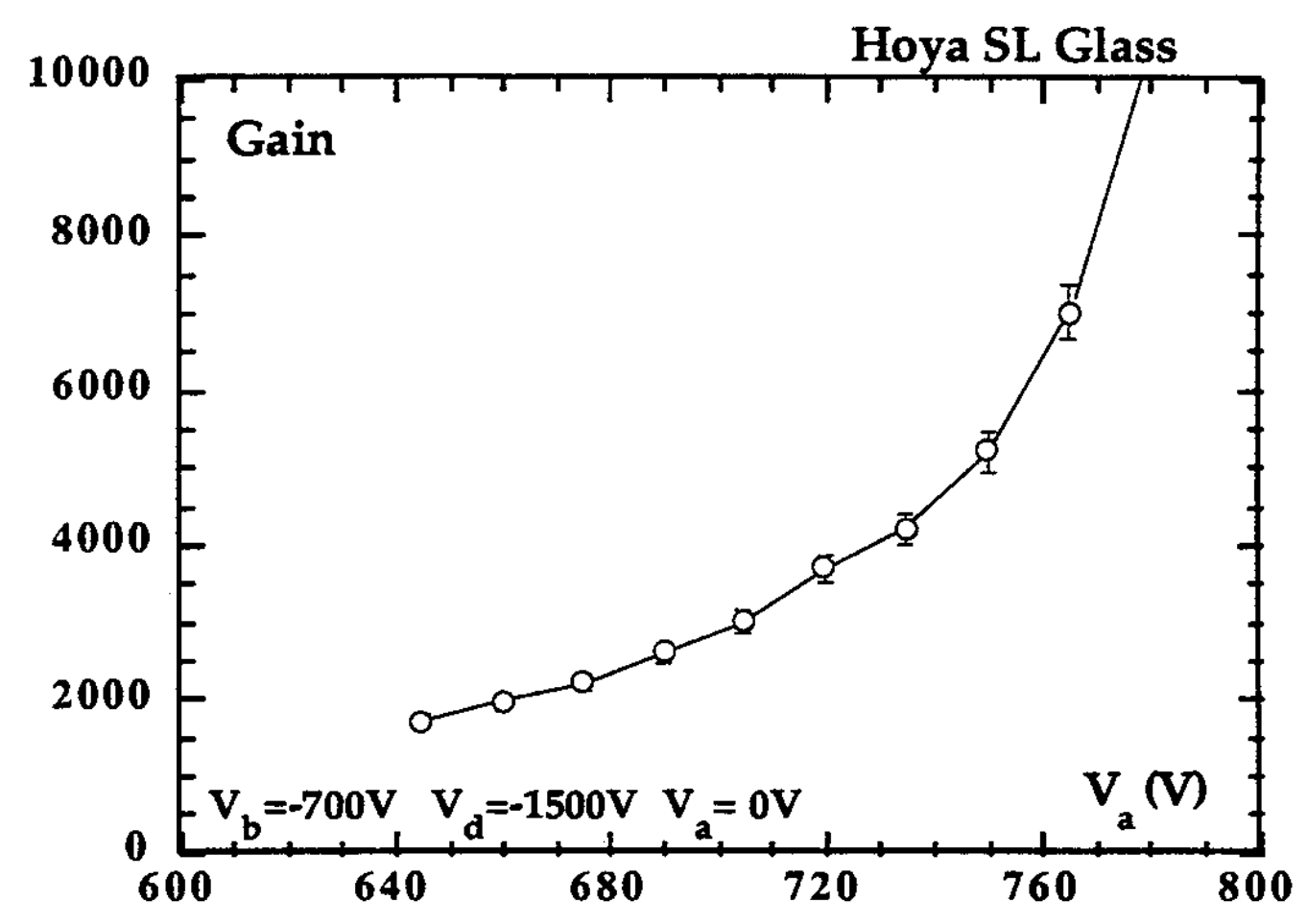

Fig. 3

Hoya SL Glass

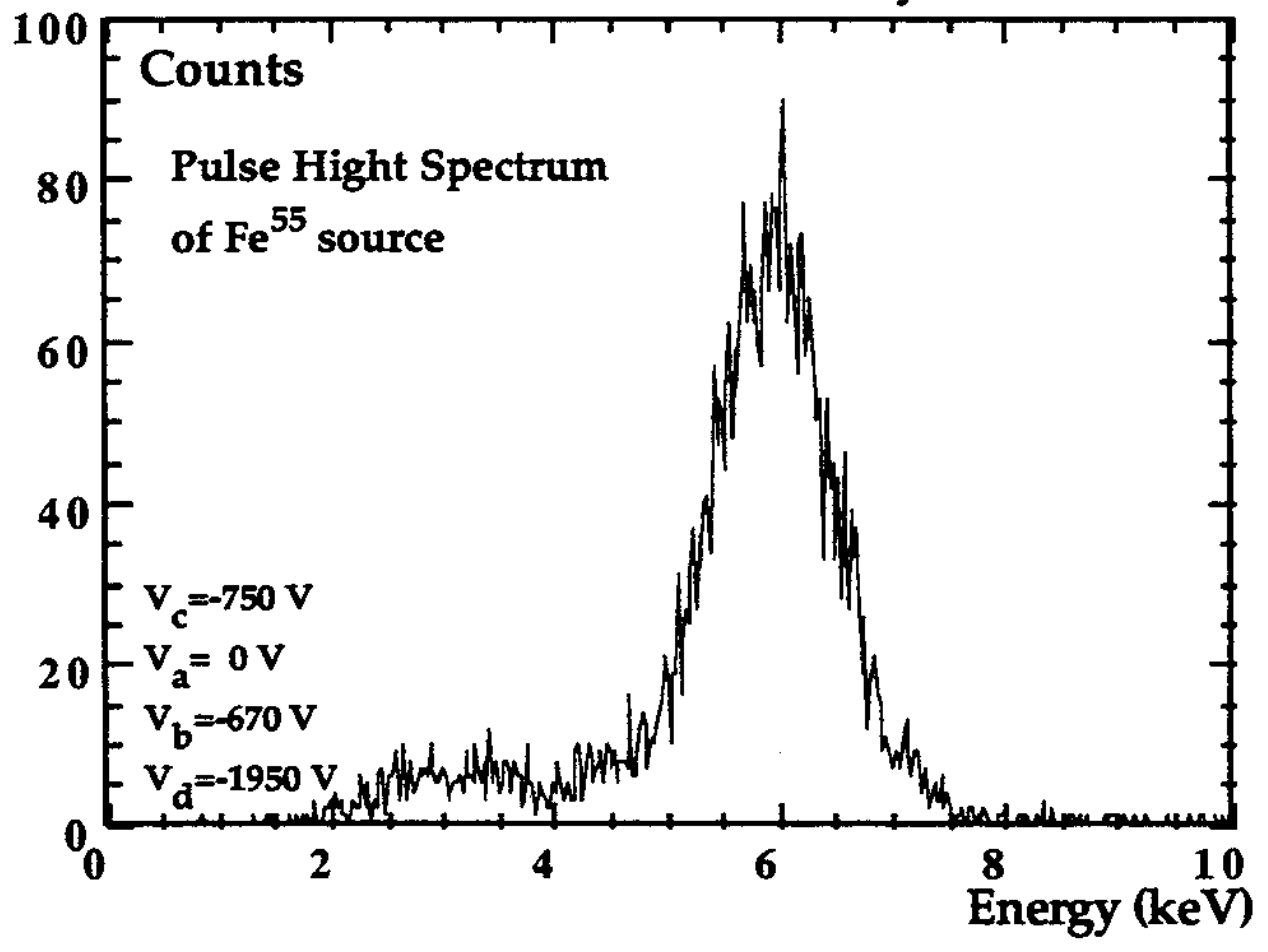

Fig. 4 
Hoya SL Glass

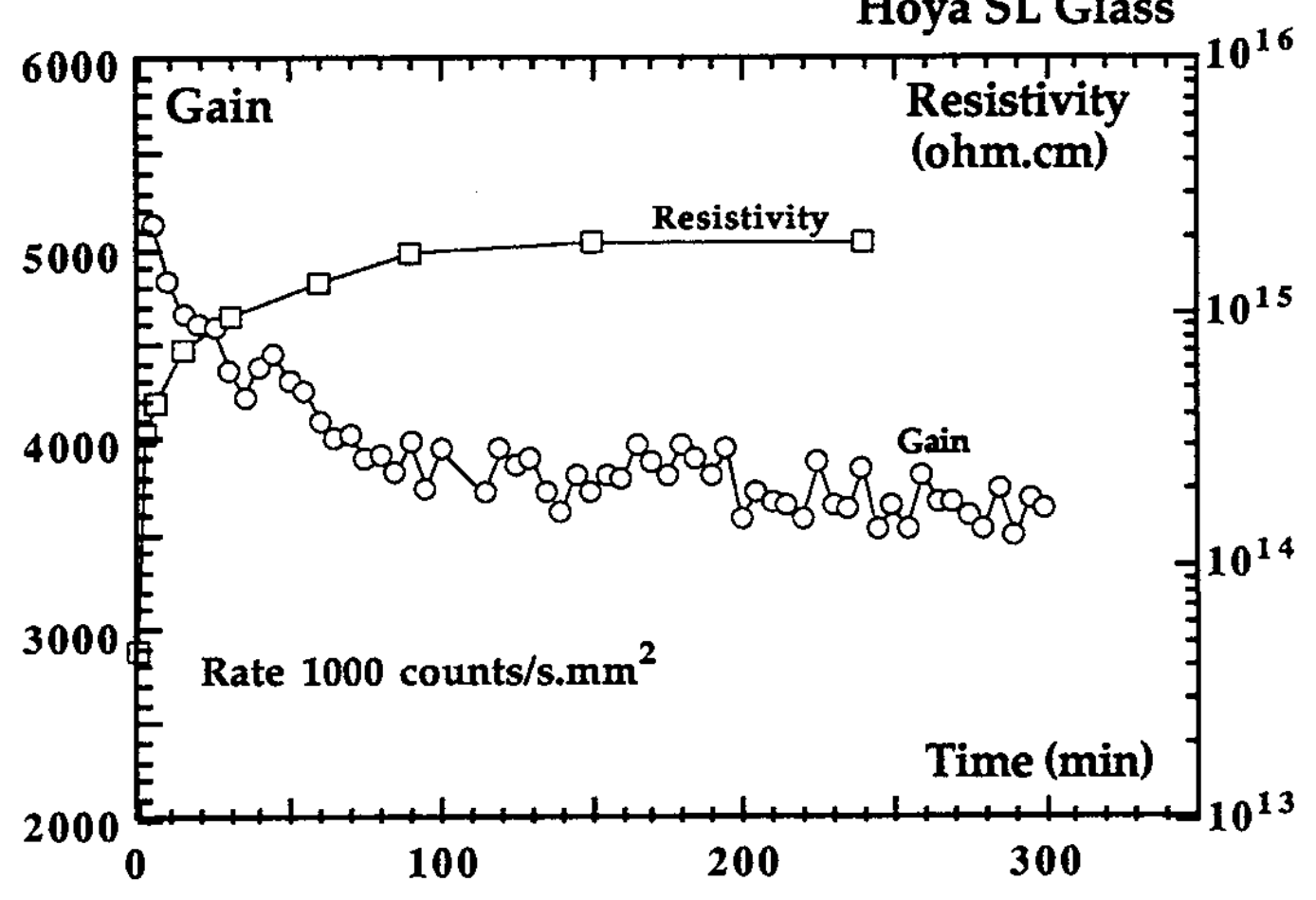

Fig. 5

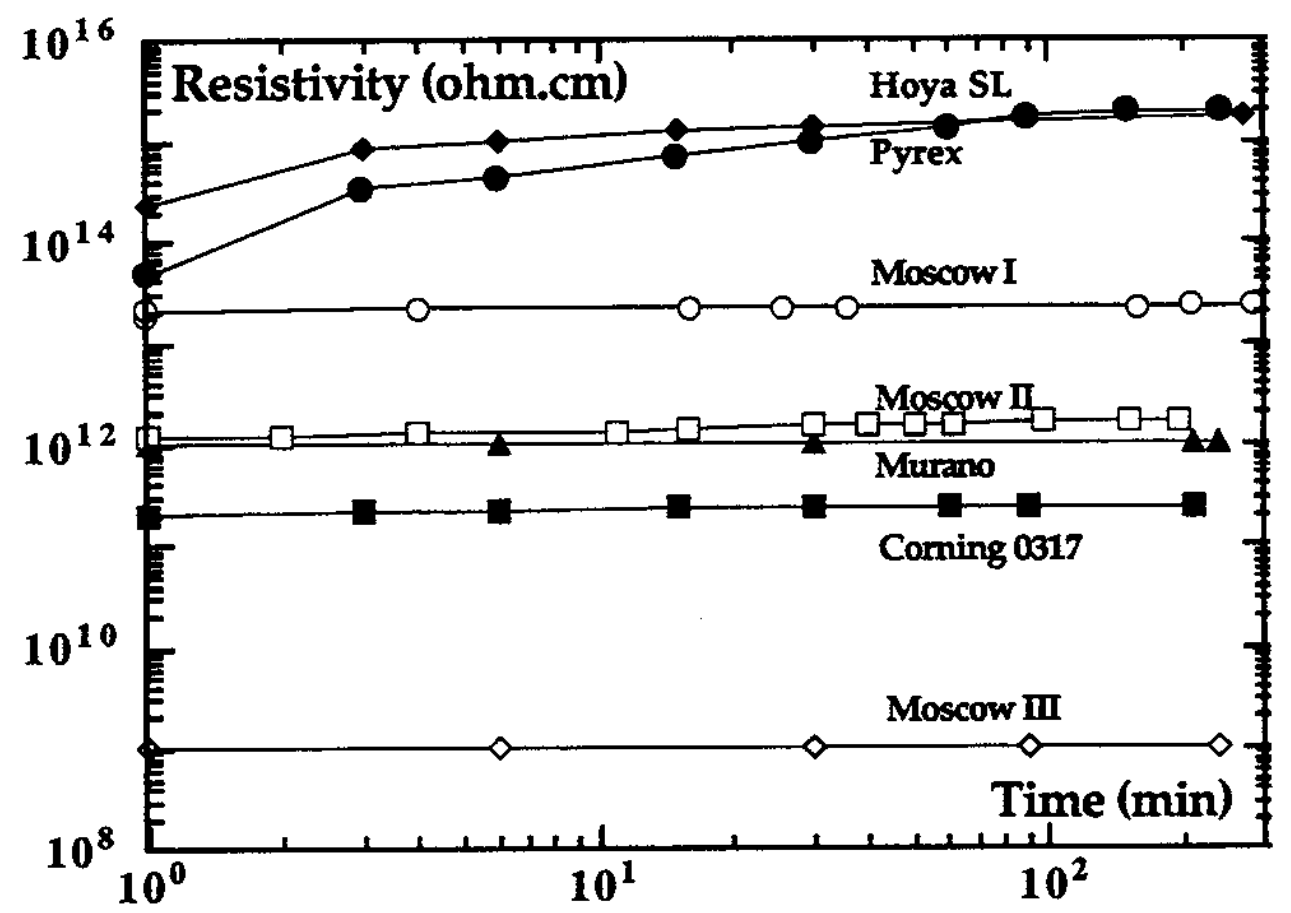

Fig. 6 


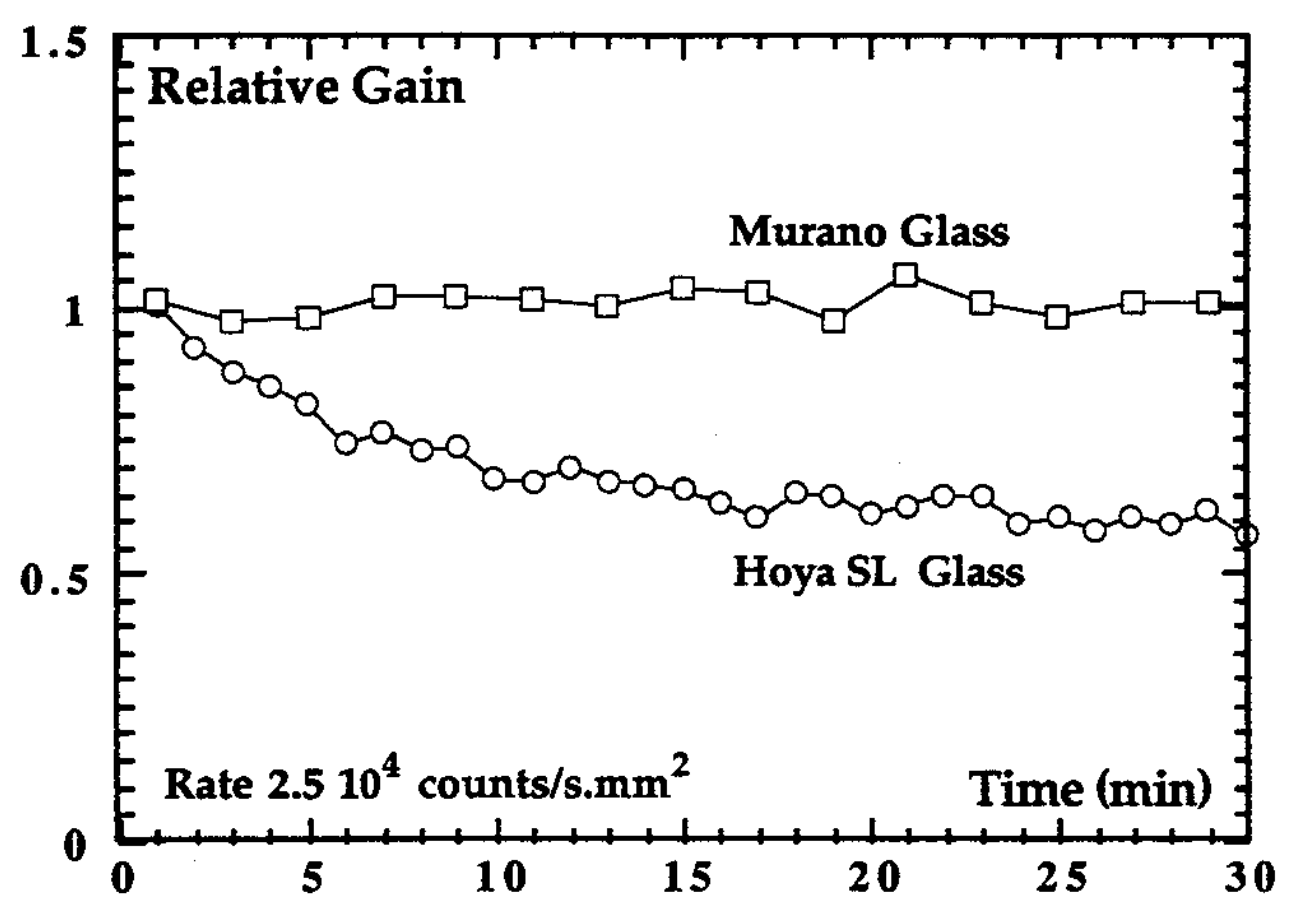

Fig. 7

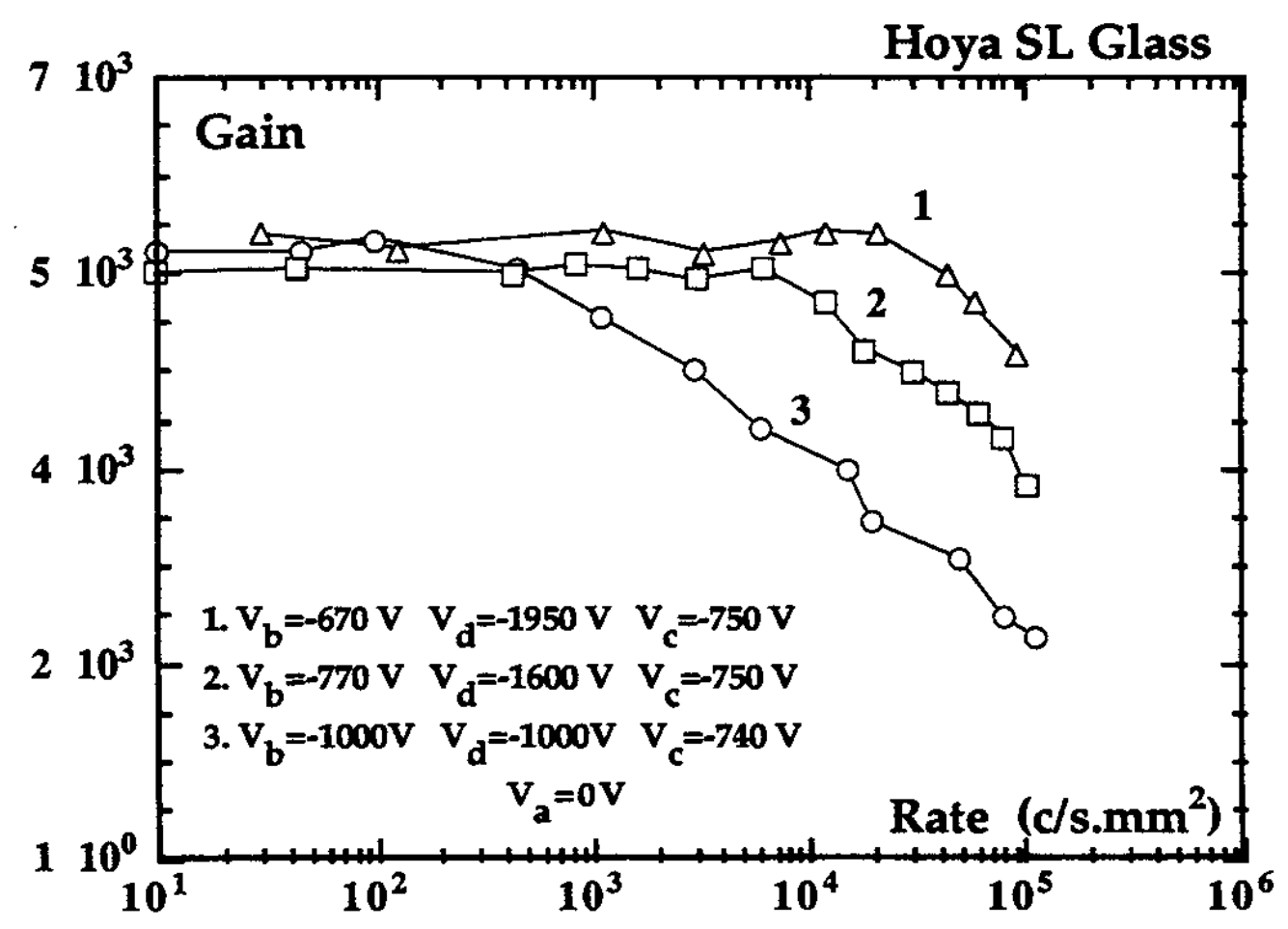

Fig. 8 


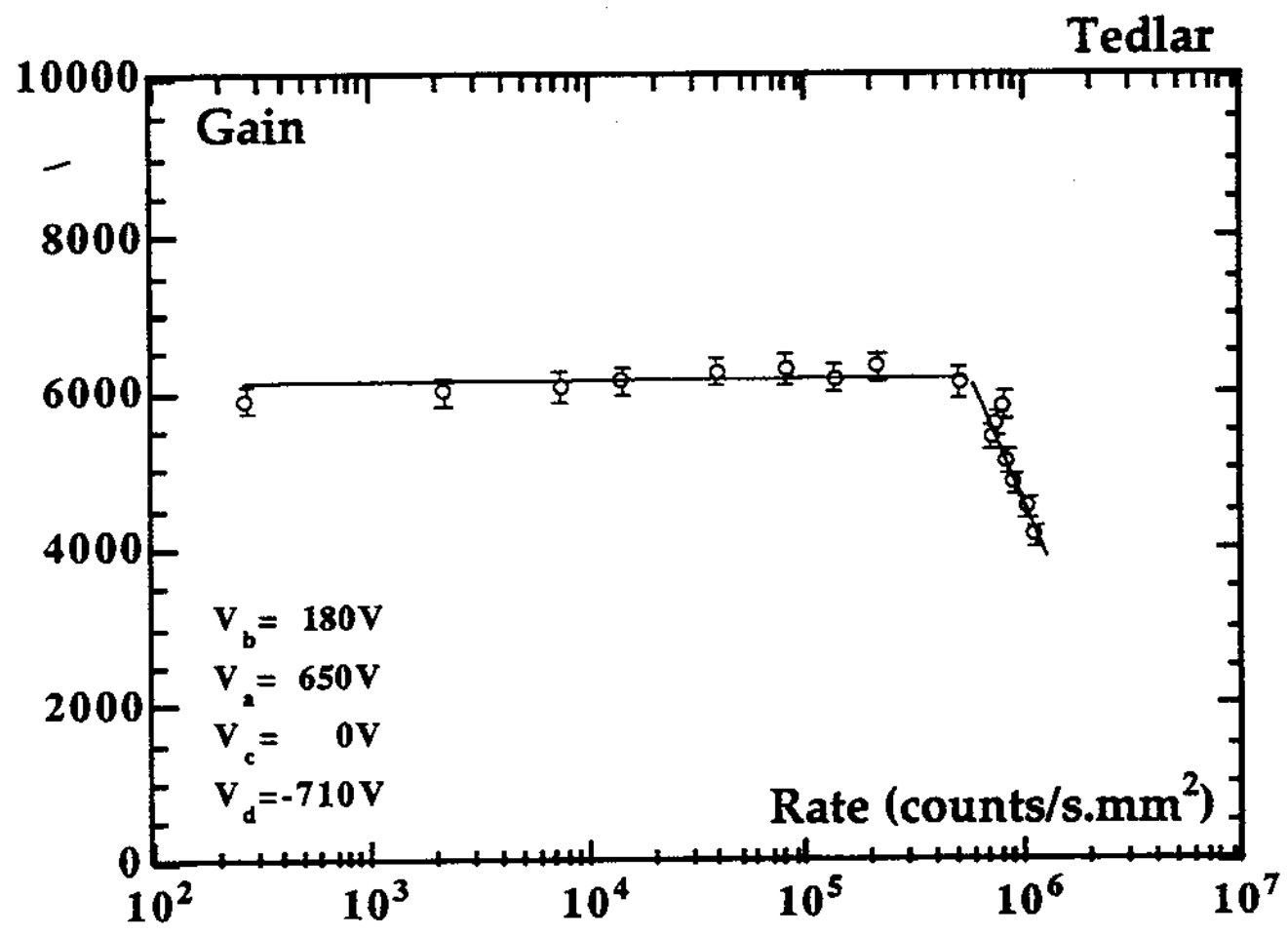

Fig. 9

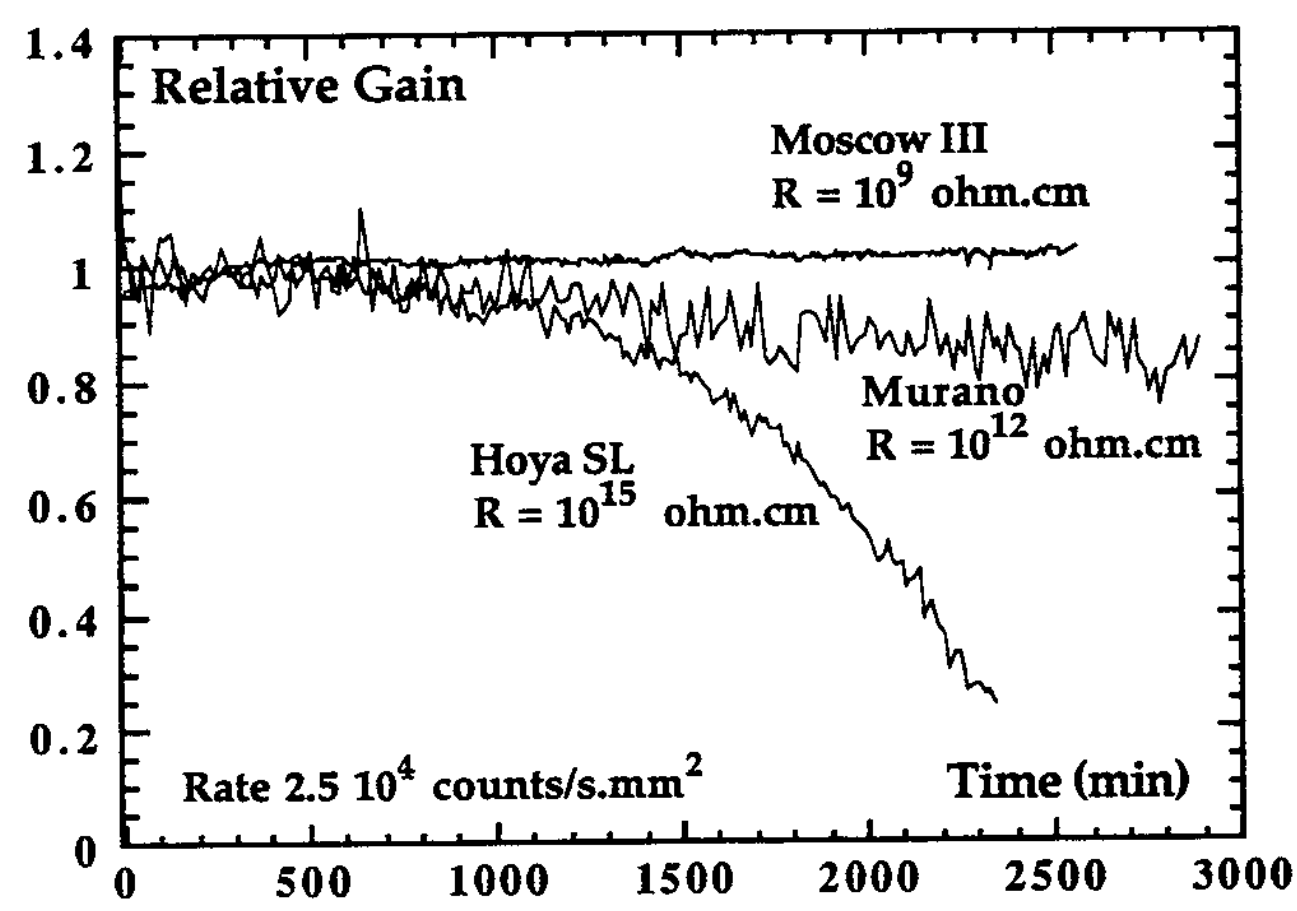

Fig. 10 


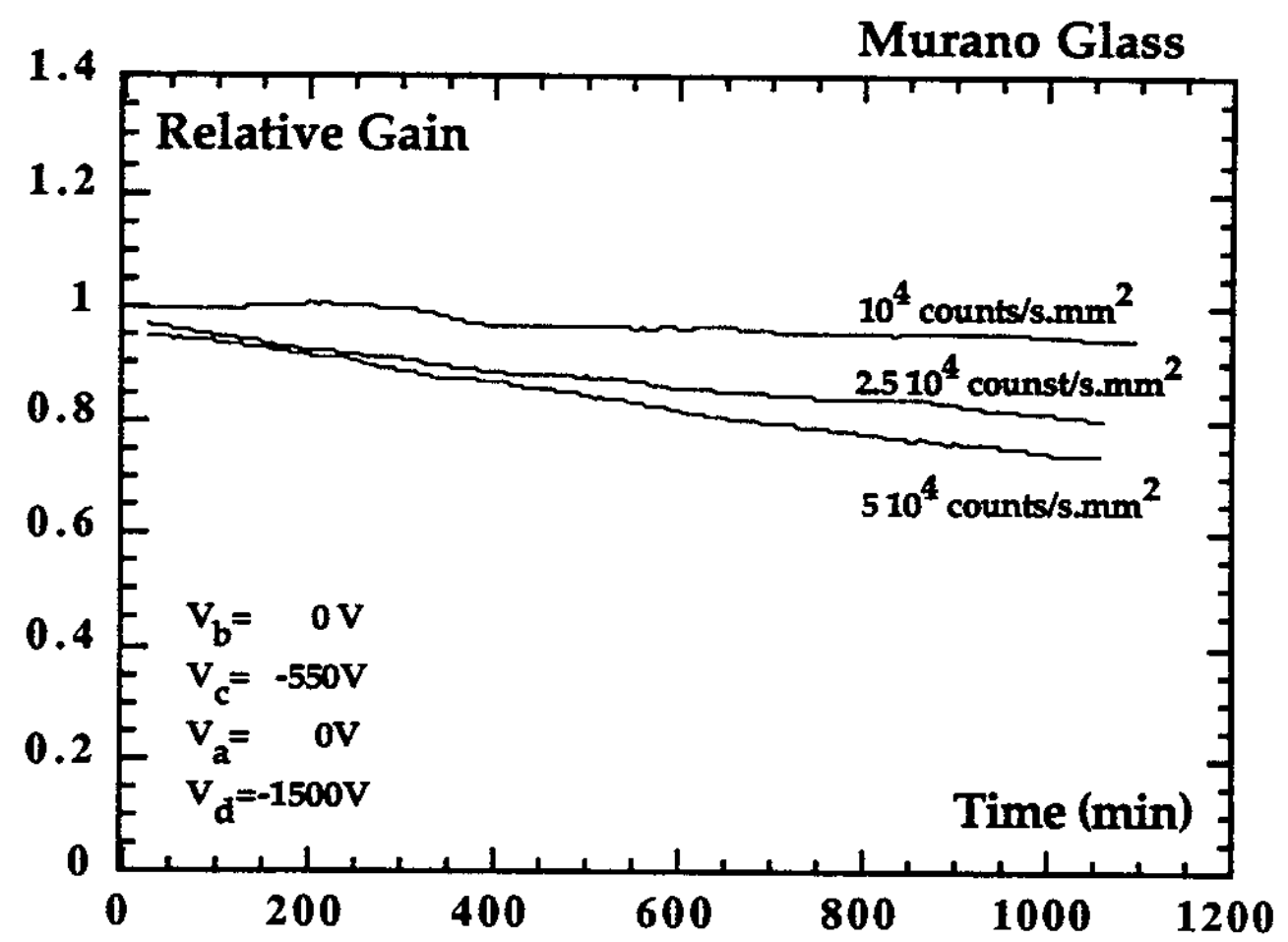

Fig. 11

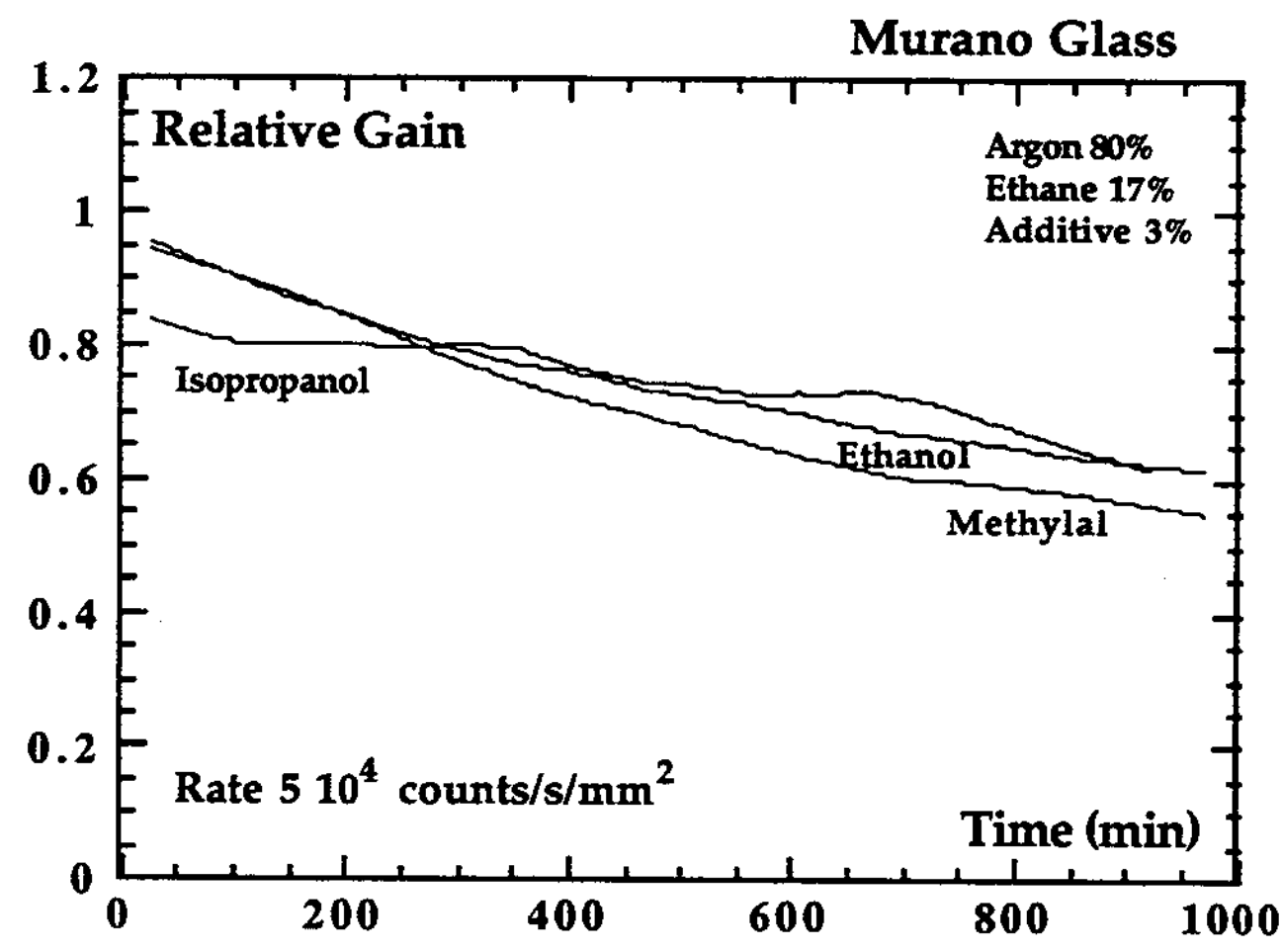

Fig. 12 


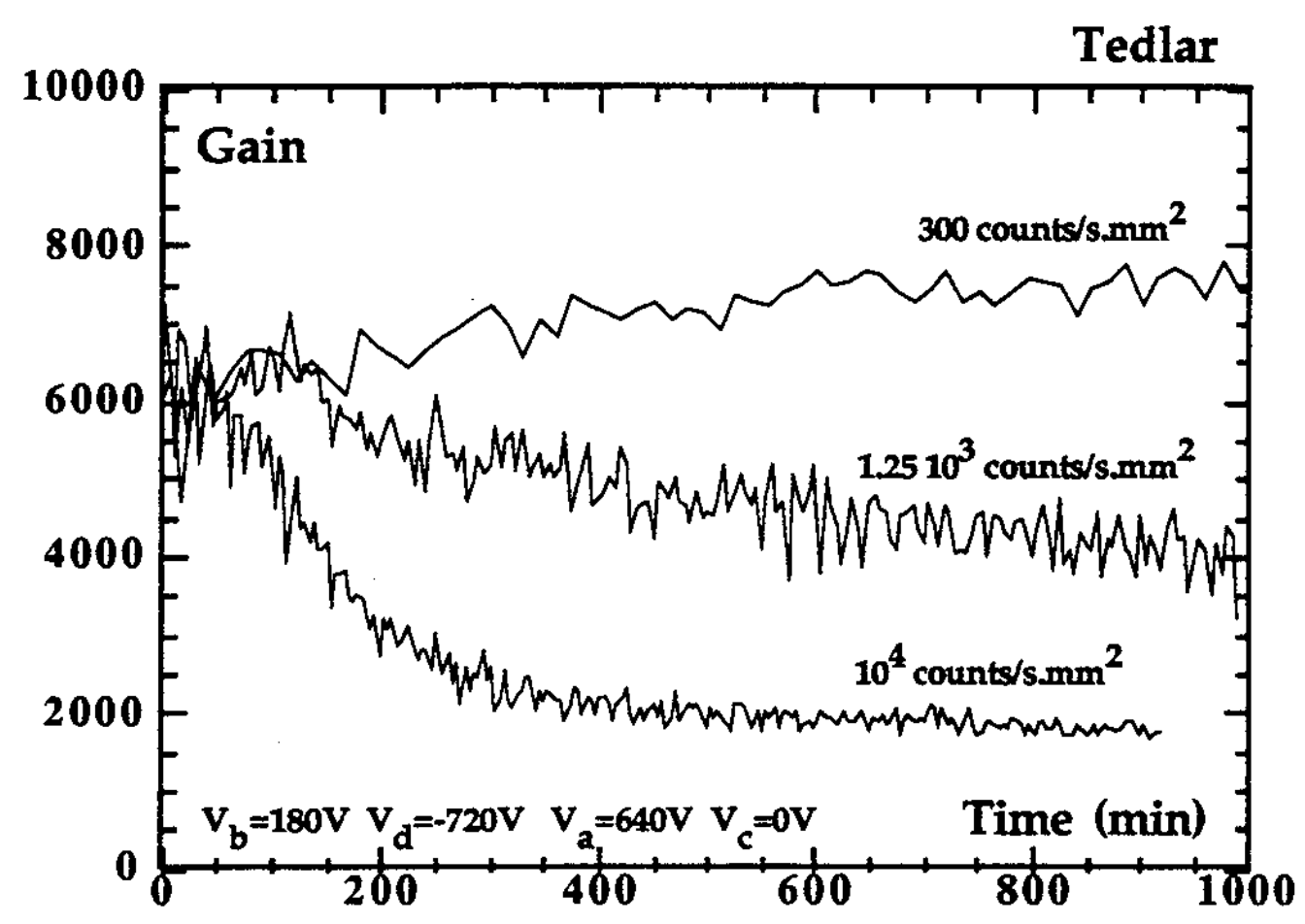

Fig. 13

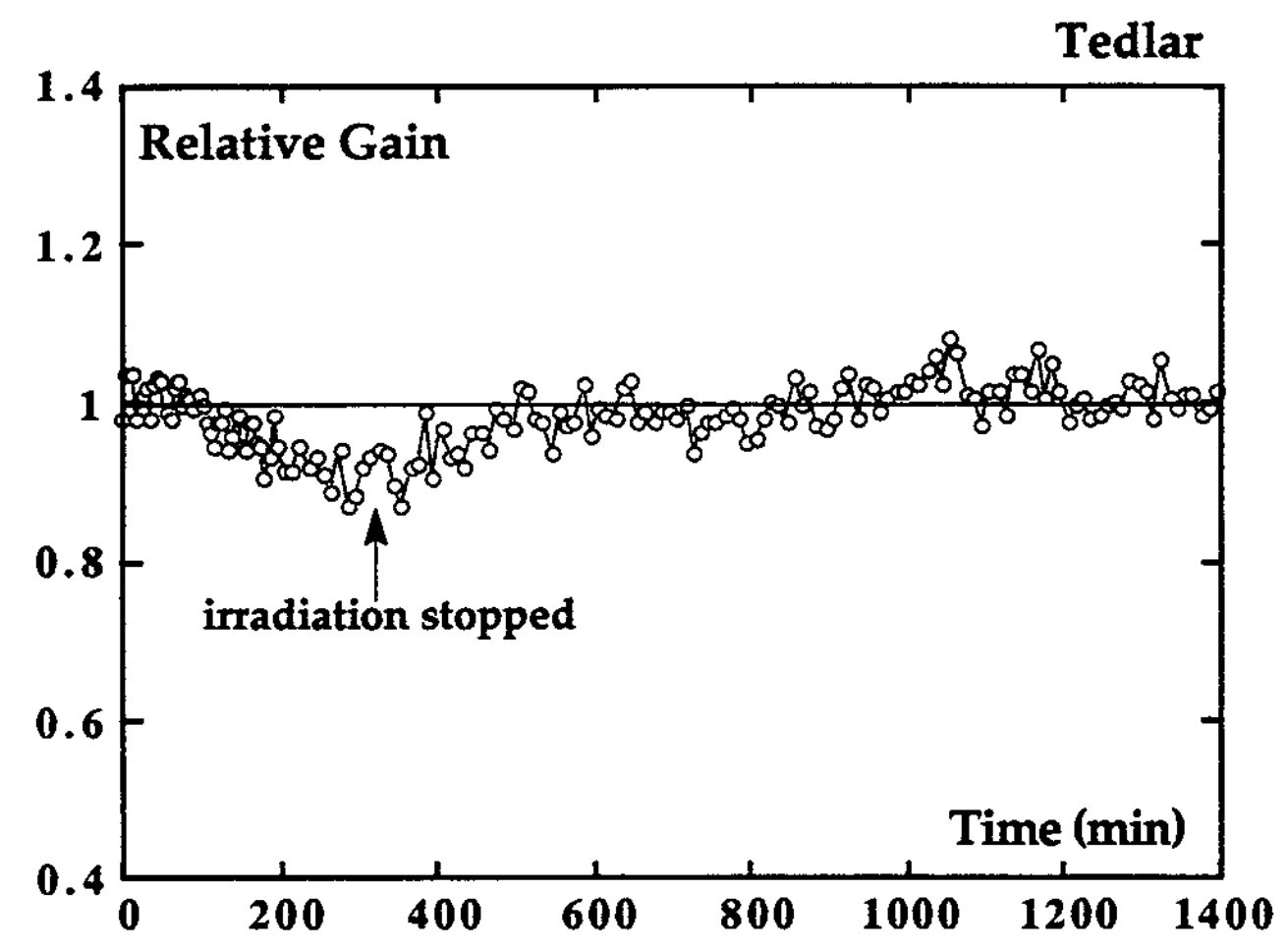

Fig. 14 